\title{
EFFECT OF CHINESE MEDICAL HERBS-HUIRU YIZENG YIHAO ON HYPERPROLACTINEMIA AND HYPERPLASIA OF MAMMARY \\ GLAND IN MICE
}

\section{Xiong Wang, Yong-gang Chen, Li Ma, Zhi-hui Li, Ju-yi Li, Xin-guo Liu, Ji-li Zou, Jin-hu Wu ${ }^{*}$}

\author{
(The Department of Pharmacy, The Third Hospitai of Wuhan, Wuhan,China. \\ ${ }^{*}$ No. 241 Peng Liu Yang Road, Wuhan 430060, Hubei Province, China) \\ *E-mail: wangxiong-133494@163.com
}

\begin{abstract}
The study investigated the pharmacodynamism and mechanism of Chinese medicinal formula-Huiru Yizeng Yihao (NO.1 HRYZ) on the model rats of hyperpro-lactinemia and the model rats of hyperplasia of mammary gland (HMG), and studied the internal connection between hyperprolactinemia and HMG.. The hyperprolactinemia rat models were established by injecting metoclopramide dihydrochloride in the back of rats. The model rat of HMG was prepared by injecting estradiol in the thigh muscle of the rats and progesterone consecutively, while the tails of rats were clipped with tongs. Rats were treated with either NO.1 HRYZ or positive control drugs for four weeks. The concentrations of sex hormone in rat serum were examined using ELISA kits, and the morphology of mammary gland tissue in all group rats was observed with microscope. NO.1 HRYZ significantly decreased prolactin (PRL) and increased estradiol $\left(\mathrm{E}_{2}\right)$, progesterone $(\mathrm{P})$, follicle stimulating hormone (FSH), luteinizing hormone ( $\mathrm{LH})$ concentrations of hyperprolactinemia rats. It decreased $\mathrm{E}_{2}, \mathrm{PRL}, \mathrm{FSH}$, gonadotropin-releasing hormone (GnRH), 5-hydroxytryptamine (5-HT) and increased P concentrations of HMG rat. It also eliminated hyperplasia of lobules and gland alveolus compared with the model group. Treatment with NO.1 HRYZ could significantly regulate the sex hormone disorder of hyperprolactinemia and HMG rat models, and could eliminate the formation of HMG. Hyperprolactinemia was closely correlated with HMG, and hyperprolactinemia promoted the formation of HMG.
\end{abstract}

Key words: NO.1 HRYZ; hyperprolactinemia; HMG; sex hormone; morphology of mammary gland

\section{Introduction}

Hyperprolactinemia, which is one of the most common endocrine disorders of the hypothalamus-pituitary axis (PRL $>25 \mathrm{ng} / \mathrm{ml}$ ) in young women, is associated with galactorrhoea and ovulatory dysfunction that results in menstrual irregularities and barren (Lee et al., 2012). Hyperprolactinemia can occur at any age, and the prevalence varies from $0.4 \%$ in the normal adult population to as high as $9-17 \%$ in women with menstrual problems such as amenorrhoea or polycystic ovarian syndrome (Biller et al., 1999; Greer et al., 1980). Typical examples which induce hyperprolactinemia are hypothalamus-pituitary lesions, pituitary tumour, severe liver or kidney disease, neuritis or irritations of the spinal cord, depression or other physiological factors such as pregnancy and lactation (Gerhard, 2012; Yu et al.,1998.). HMG is a kind of pathological hyperplasia of lobules of mammary gland induced by the balance disorder of oestrogen and progesterone( Bennett et al., 1990), which were controlled and secreted abnormally by hypothalamus-pituitary-gonadal axis (seen in Figure 1). HMG is related to menstrual cycle, breast-feeding, occupation, abuse of sex hormone drugs, diet and mental pressure (Xu, 2011; Jia, 2011.). The number of patients with HMG is increasing in recent years, and it has severe cancerous tendencies, and may have been mixed and covered with early breast cancer (Wang et al., 2011). In clinical, some hyperprolactinemia patients were found accompanied with HMG (Lu, 2004), but the internal correlation between 

http://dx.doi.org/10.4314/ajtcam.v10i4.5

hyperprolactinemia and HMG is not clear yet.

Chemical drugs are used in treating hyperprolactinemia or HMG, but they always bring many side effects such as menstrual disorder, and the relapse rate is very high. Bromocriptine and cabergoline are effective in curing hyperprolactinemia, but $12 \%$ of patients cannot endure bromocriptine (Webster et al., 1994), and they are expensive

(Mah and Webster, 2002). Hormone drugs such as progesterone or testosterone propionate can fight against increased oestrogen to regulate the balance of hormone in curing HMG (Fallois et al., 1995). Tan (Tan et al., 2003) found that tamoxifen could significantly relieve the symptom of HMG, but it still had some side effects such as gastrointestinal reaction, vomit or diarrhoea, menstrual disorder, neuropsychiatric symptom, headache or vertigo. Hence, patients often seek herbal therapies outside of the traditional approaches. Chinese medicinal formula No.1 HRYZ has the effect of regulating endocrine disorder. It is widely used in China for the treatment of hyperprolactinemia and HMG and has produced a favourable effect.

The objective of the study is to investigate the pharmacodynamic and mechanism of No.1 HRYZ in model rats of hyperprolactinemia and HMG, while also studying the correlation between hyperprolactinemia and HMG preliminary.

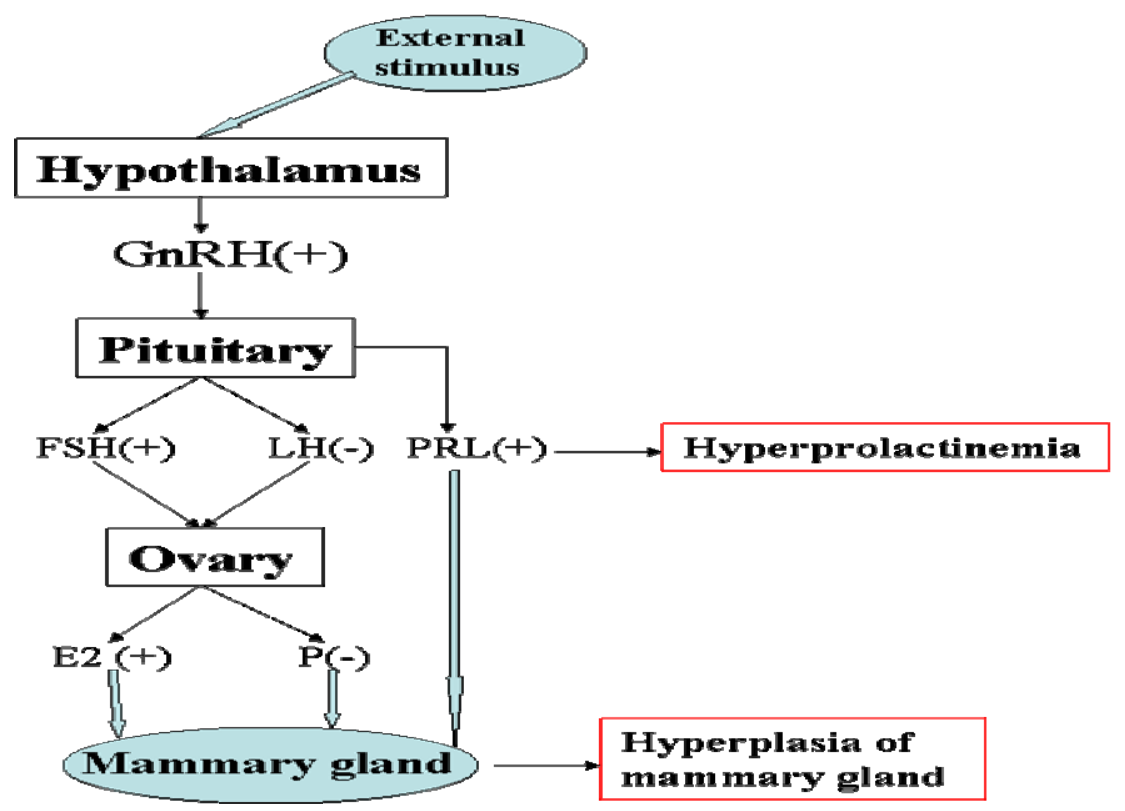

Figure 1: The occurrence mechanism of hyperprolactinemia and HMG

\section{Materials and methods}

\section{Materials}

Female Wistar rats weighing 200-220g were obtained from Hubei Center for Disease Control and Prevention, Wuhan, Hubei. The animals had free access to food and water, and were allowed to acclimatise for at least one week before use. All experiments conformed to the guidelines of the "Principles of Laboratory Animal Care” (NIH publication No.80-23, revised 1996) and the legislation of the People’s Republic of China for the use and care of laboratory animals. Metoclopramide was obtained from Xuzhou Laien Pharmaceutical Co., Ltd. Bromocriptine methanesulfonate was obtained from Novartis Pharma Schweiz AG. Tamoxifen, a nonsteroidal anti-oestrogen, anticancer drug, was purchased from Ningbo Tianheng Pharmaceutical Co., Ltd. Rupisanjie caspule, a kind of frequently-used Chinese patent drug which is embodied in Chinese Pharmacopoeia (2010 edition) and has the curative effect for curing HMG in China, which was purchased from Shanxi Bailu Pharmaceutical Co., Ltd., was used as positive control for curing HMG. All other reagents and solvents were of analytical grade.

NO.1 HRYZ formula, composed of Mai Ya (Fructus Hordei Germinatus), Xia Ku Cao (Prunella vulgaris L.), Bai Shao (Paeonia lactiflora Pallas), Zhe Bei Mu (Fritillaria thunbergii Miq.), Ru Xiang (Boswellia carterii Birdw.),Yu Jin(Curcuma 

http://dx.doi.org/10.4314/ajtcam.v10i4.5

aromatica Salisb.), Dan Shen (Salvia miltiorrhiza Bge.), Bai Jie Zi (Semen Brassicae), Mu Li (Ostrea gigas thunberg) and Kun Bu (Laminaria japonica Aresch.), were provided by the third hospital of Wuhan (Wuhan, China).

\section{Preparation of model rats}

The hyperprolactinemia rat model was performed as previously described with a little modification (Lin et al., 1988). Metoclopramide dihydrochloride injection was subcutaneously injected in the back of rats according to $50 \mathrm{mg} / \mathrm{kg}$ body weight. Experiment was performed at 9:00 and 15:00 respectively every day for five days. Estradiol was injected in the thigh muscle of the rats for twenty-five days, then was progesterone administered in like manner consecutively for five days (Rao et al., 1992; Milliken et al., 2002). Meanwhile, tails of rats were clipped with tongs for thirty minutes every day. In this way, we prepared HMG rat model.

\section{Experimental Groups and Treatments}

The model rats of hyperprolactinemia were divided into six groups of ten individual groups: control, model group, positive drug bromocriptine group $\left(0.393 \mathrm{mg} \cdot \mathrm{kg}^{-1} \cdot \mathrm{d}^{-1}\right)$, model plus NO.1 HRYZ(33.1 $\left.\mathrm{g}^{\mathrm{kg}}{ }^{-1} \cdot \mathrm{d}^{-1}\right)$, plus NO.1 HRYZ (16.55 $\left.\mathrm{g} \cdot \mathrm{kg}^{-1} \cdot \mathrm{d}^{-1}\right)$, and plus NO.1 HRYZ(8.28 $\left.\mathrm{g}^{\mathrm{kg}}{ }^{-1} \cdot \mathrm{d}^{-1}\right)$. The model rats of HMG were randomly divided into eight groups of ten individuals: control, disease model, integrated disease model, integrated disease model plus NO.1 HRYZ(31.6g/kg), plus NO.1 HRYZ(15.8g/kg), plus NO.1 HRYZ(7.9 g/kg), plus positive drug tamoxifen $(1.57 \mathrm{mg} / \mathrm{kg}$ ) and plus positive drug Rupisanjie capsule $(0.5 \mathrm{~g} / \mathrm{kg})$. Each dose was dissolved in a 2-ml mixture of distilled water and administered intragastrally (i.g.) using a 5-ml syringe with a 4-cm long gavage needle through the mouth to the stomach. The initial dosage was based on body surface area and calculated from the daily human NO.1 HRYZ clinical dosage (FDA,2002). Control, hyperprolactinemia model or HMG disease and integrated disease model rats received $2 \mathrm{ml}$ of water. All group mice were intragastrically administrated for thirty days.

\section{Blood serum collection and ELISA}

At the end of thirty days, $\mathrm{E}_{2}, \mathrm{P}, \mathrm{PRL}, \mathrm{FSH}, \mathrm{LH}$ in the hyperprolactinemia rat serum and $\mathrm{E}_{2}, \mathrm{P}, \mathrm{PRL}, \mathrm{FSH}, \mathrm{LH}, \mathrm{GnRH}$, 5-HT in the HMG rat serum were measured. All measurements were used by enzyme-linked immunosorbent assay (ELISA) kits according to the procedures recommended by the manufacturer (Shan hailichen, China).

\section{Histological study}

Tissues of mammary gland from each group were fixed in $10 \%$ buffered formalin, embedded in paraffin, sectioned into $4 \mu \mathrm{m}$ thickness, stained with Haematoxylin-Eosin

(H-E) and Masson-Trichrome (M-T) and examined using optical microscopy. The severity of mammary gland hyperplasia was based on four parameters (hyperplasia of gland alveolus, lobule, shape and thickness of vessel and secretion).

\section{Acute toxicities of NO.1 HRYZ in mice}

Kunming mice (18-22 g) were housed in cages at $22^{\circ} \mathrm{C}$. Prior to the experiments, they were starved overnight with free access to water. NO.1 HRYZ decoction was prepared into freeze-dried powder (1g freeze-dried powder was equal to $16 \mathrm{~g}$ NO.1 HRYZ crude drug). Test one: ten mice were divided into male and female half and given 43.1g/kg (200 times of adult dosage) or $34.9 \mathrm{~g} / \mathrm{kg}$ (162 times of adult dosage) freeze-dried powder every day for seven days. We observed the death after administration of twenty-four hours and seven days. Test two: twenty mice were divided into male and female half and given 

http://dx.doi.org/10.4314/ajtcam.v10i4.5

$38.8 \mathrm{~g} / \mathrm{kg}$ (180 times of adult dosage) freeze-dried powder every day for fourteen days. We observed immediate toxicity reaction after administration and survival situation of mice in fourteen days.

\section{Statistical analysis}

Values are expressed as means \pm SEM. Multiple group comparisons were performed using one-way analysis of variance (ANOVA) followed by Dunnett's test to detect intergroup differences. $P<0.05$ was considered significant in all cases.

\section{Results}

Effect of NO.1 HRYZ on E, P, PRL, FSH and LH levels in hyperprolactinemia rat serum

Hyperprolactinemia model rat significantly increased serum PRL concentration and decreased serum $\mathrm{E}_{2}$, P, FSH and LH concentrations $(P<0.05$ vs. blank control). Such an increased PRL was significantly attenuated by treatment with bromocriptine, high-dose and middle-dose NO.1 HRYZ after one month administration $(P<0.05$ vs. the "model” control). Bromocriptine raised decreased $\mathrm{E}_{2}, \mathrm{P}$ significantly ( $P<0.01$ vs. the "model” control). $\mathrm{E}_{2}$ of high-dose NO.1 HRYZ group increased remarkably ( $P<0.05$ vs. the "model” control), and so middle-dose NO.1 HRYZ group $(P<0.01$ vs. the "model” control). Decreased P was increased by high-dose, middle-dose and low-dose NO.1 HRYZ $(P<0.05$ vs. the "model” control). Both of FSH ( $P<0.01$ vs. the "model” control) and LH ( $P<0.05$ vs. the "model” control) increased only in high-dose and middle-dose NO.1 HRYZ group (Figure 2-6). These illustrated that NO.1 HRYZ had obvious curative effect in treating hyperprolactinemia, which was even better than positive drug bromocriptine.

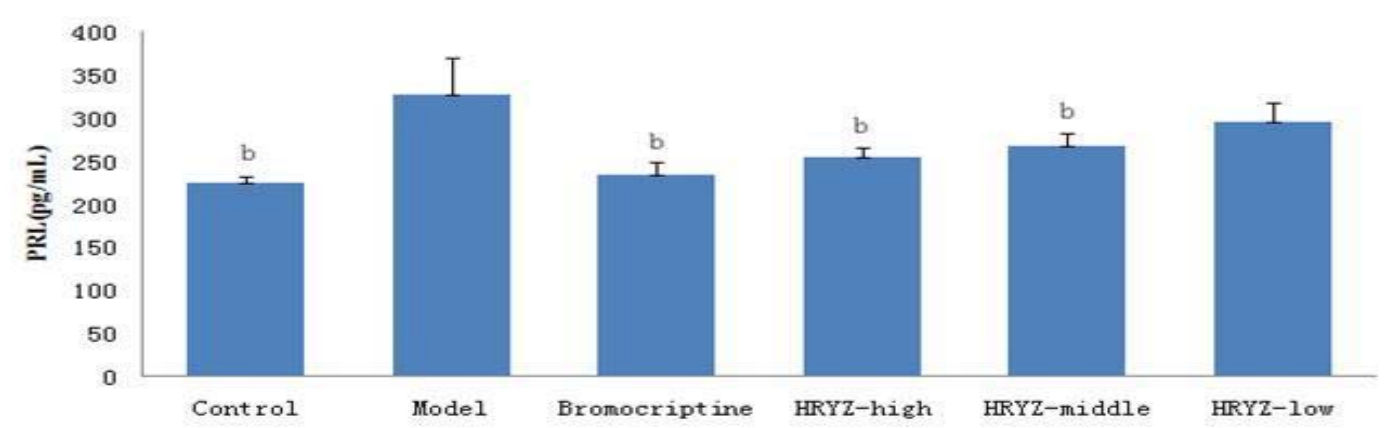

Figure 2: Serum PRL concentration in different groups ${ }^{\mathrm{b}} . P<0.05,{ }^{\mathrm{c}} P<0.01$ vs. model group

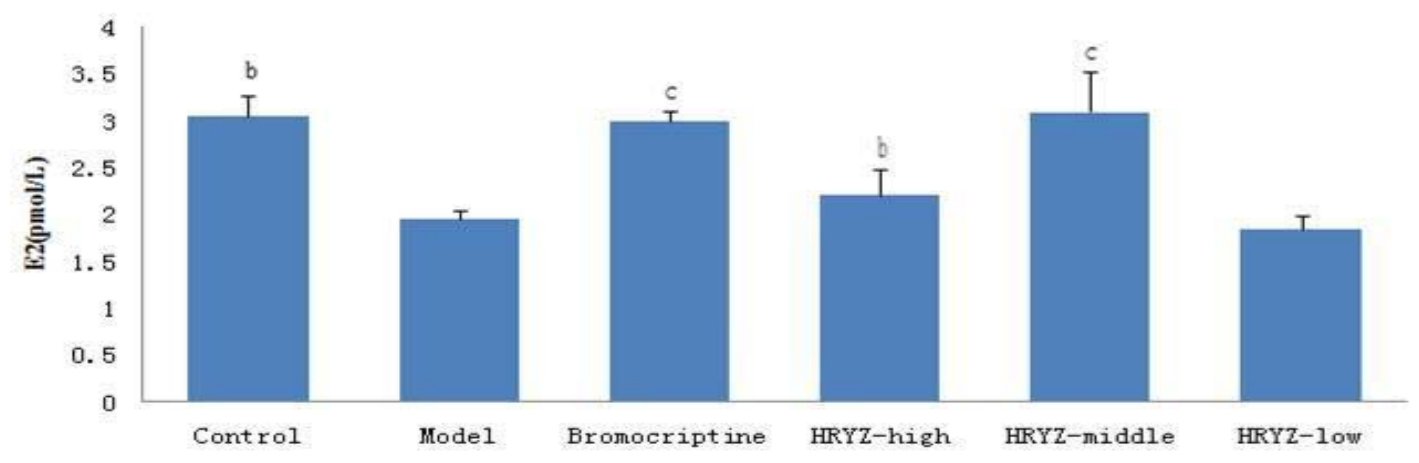

Figure 3: Serum E2 concentration in different groups ${ }^{\mathrm{b}} . P<0.05^{\mathrm{c}}, P<0.01$ vs. model group 


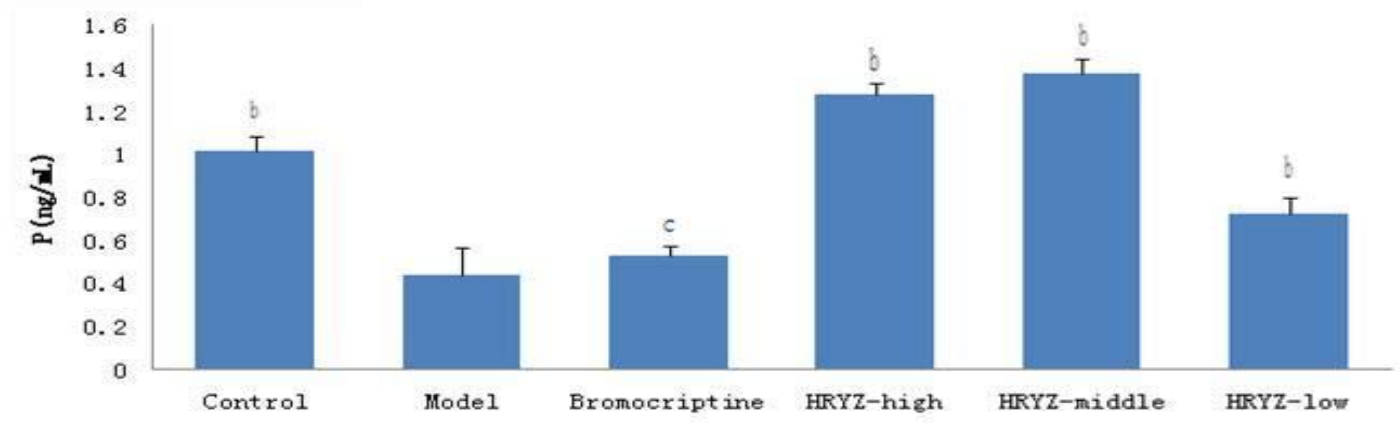

Figure 4: Serum $P$ concentration in different groups ${ }^{\mathrm{b}} . P<0.05^{\mathrm{c}}, P<0.01$ vs. model group

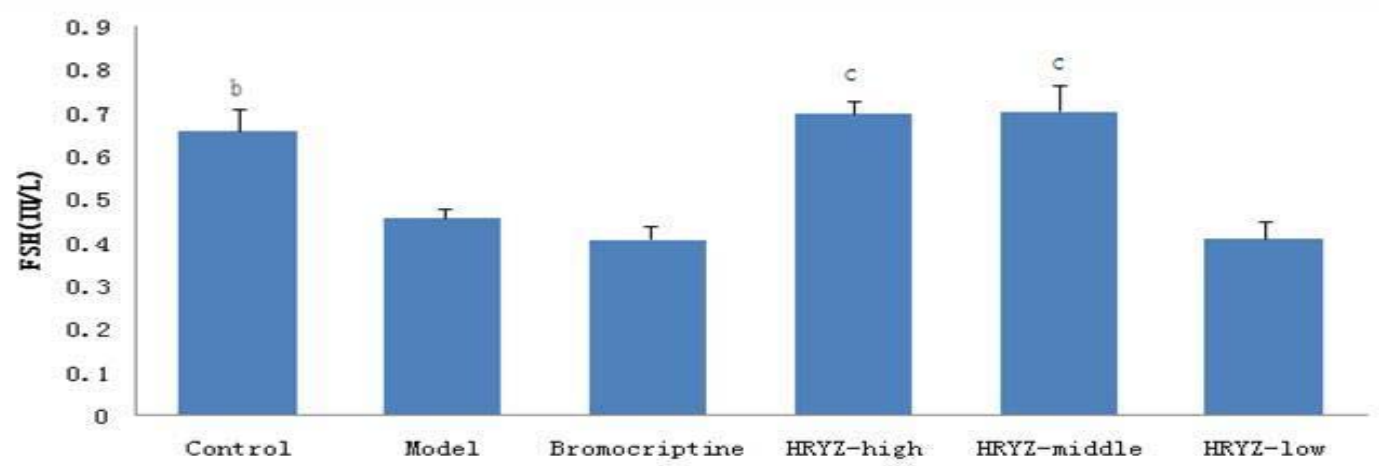

Figure 5: Serum FSH concentration in different groups ${ }^{\mathrm{b}} . P<0.05^{\mathrm{c}}, P<0.01$ vs. model group

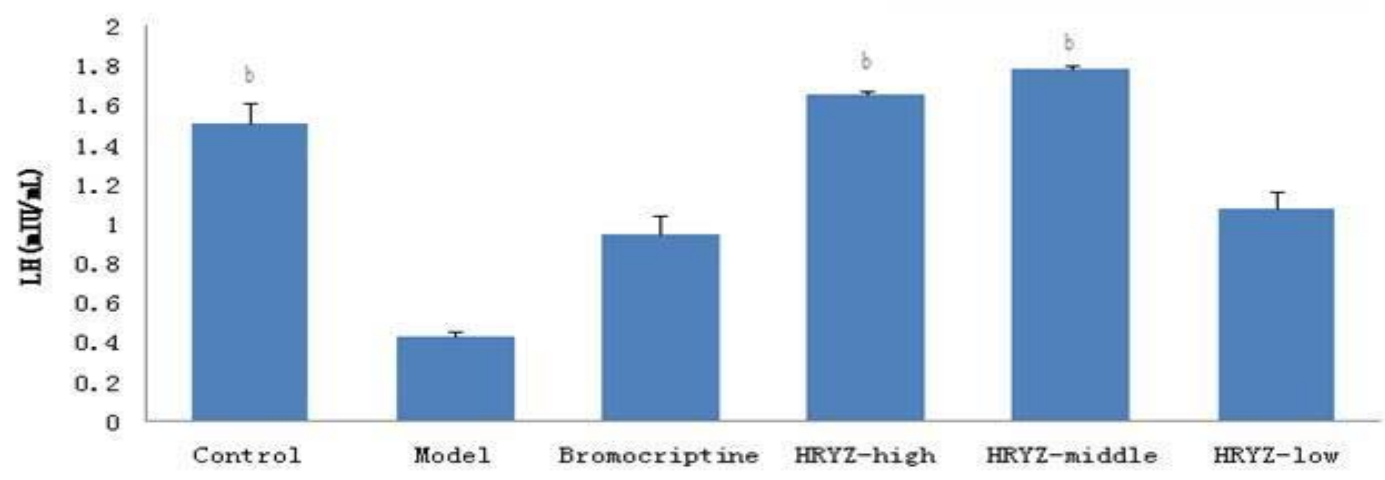

Figure 6: Serum LH concentration in different groups ${ }^{\mathrm{b}} . P<0.05^{\mathrm{c}}, P<0.01$ vs. model group

\section{Effect of NO.1 HRYZ on pathomorphology of mammary gland tissue in the}

\section{hyperprolactinemia model rats}

Compared with control group (Figure 7A), slight hyperplasia of lobules and gland alveolus, and a few secretions were found in the mammary gland of hyperprolactinemia model rats (Figure 7B). In bromocriptine-treated group at week 4, the hyperplasia of lobules and gland alveolus of mammary gland lessened, secretion and lymphocyte decreased in intracavitary (Figure 7C). The mammary gland of groups given high-dose NO.1 HRYZ and middle-dose NO.1 HRYZ recovered well. There was no hyperplasia in gland alveolus and lobules of mammary gland, secretion and lymphocyte (Figure 7D and E). A few gland alveolus and lobules hyperplasia were seen in the low-dose NO.1 HRYZ yet (Figure 7F). This illustrated that NO.1 HRYZ could inhibit the hyperplasia of mammary gland in hyperprolactinemia model rats effectively. 

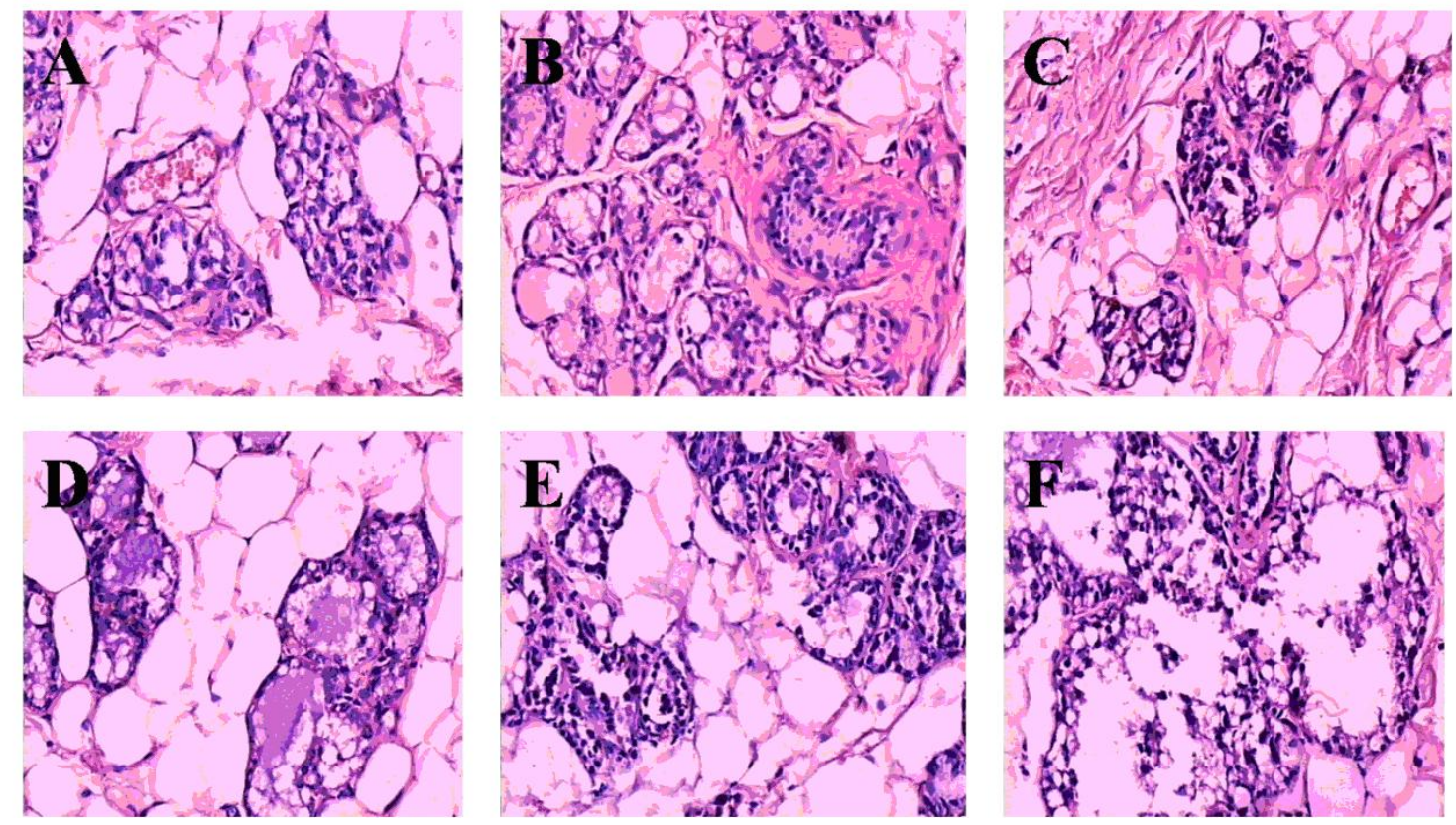

Figure 7: Morphology of mammary gland tissue was observed by microscope

(Original magnification, 200×); (A) blank controls; (B) hyperprolactinemia model group; (C) bromocriptine group; (D) high-dose HRYZ; (E) middle-dose HRYZ; (F) low-dose HRYZ

\section{Effect of NO.1 HRYZ on E, P, PRL, FSH, LH, GnRH and 5-HT levels in}

\section{HMG model rats}

HMG disease model and integrated disease model rats had significantly increased plasma $\mathrm{E}_{2}$ concentration $(P<0.01$ vs. blank control; Figure 8). Such a response was significantly attenuated by treatment with tamoxifen and Rupisanjie $(P<$ 0.05 vs. integrated disease model group for both). High-dose, middle-dose and low-dose NO.1 HRYZ treatment also remarkably decreased $\mathrm{E}_{2}$ concentration $(P<0.01)$.

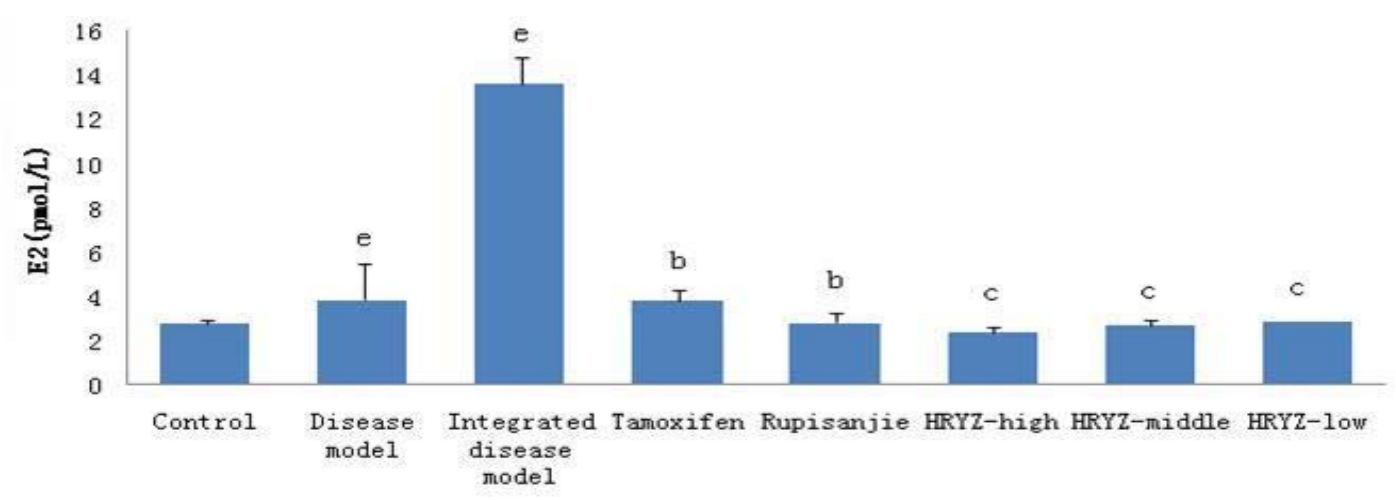

Figure 8: Serum E2 concentration in different groups ${ }^{\mathrm{d}}$. $P<0.05^{\mathrm{e}}, P<0.01$ vs. control group ${ }^{\mathrm{b}} ; P<0.05^{\mathrm{c}}, P<0.01$ vs. integrated disease model group

HMG disease model rat significantly decreased plasma P concentration ( $P<0.05$ vs. blank control; Figure 9$)$. Such a response significantly added to treatment with tamoxifen and Rupisanjie $(P<0.05$ vs. integrated disease model group for both). High-dose and middle-dose NO.1 HRYZ treatment significantly increased P concentration $(P<0.01$ vs integrated disease model group), and low-dose NO.1 HRYZ also increased P concentration $(P<0.05$ vs. integrated disease model group). 


\section{http://dx.doi.org/10.4314/ajtcam.v10i4.5}

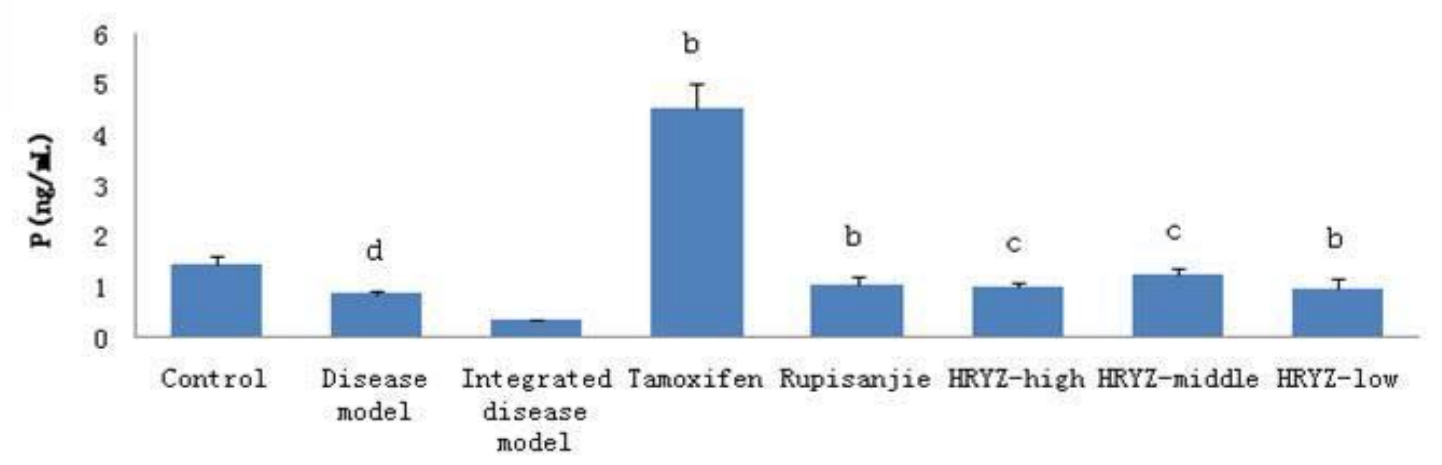

Figure 9: Serum $\mathrm{P}$ concentration in different groups ${ }^{\mathrm{d}}$. $P<0.05^{\mathrm{e}}, P<0.01$ vs. control group ${ }^{\mathrm{b}} ; P<0.05^{\mathrm{c}}, P<0.01$ vs. integrated disease model group

HMG disease model and integrated disease model rats significantly increased plasma PRL concentration $(P<0.05$ vs. blank control; Figure 10). Such a response was significantly attenuated by treatment with Rupisanjie, high-dose, middle-dose and low-dose NO.1 HRYZ treatment ( $P<0.05$ vs. integrated disease model group). Tamoxifen group had no significant difference.

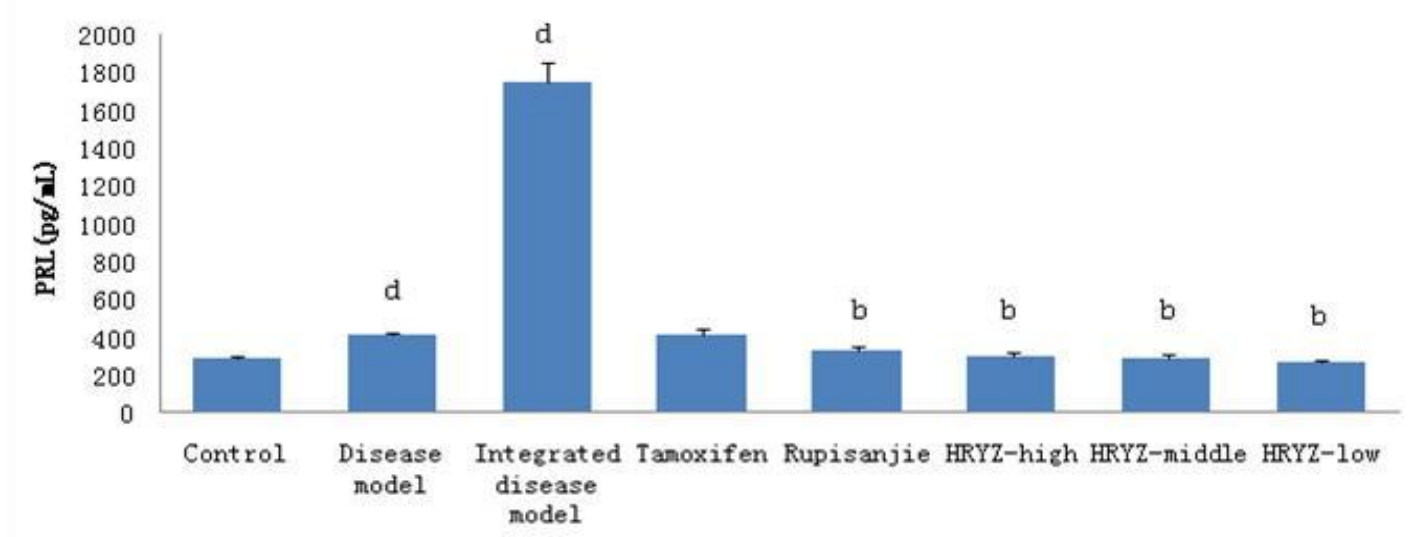

Figure 10: Serum PRL concentration in different groups ${ }^{\mathrm{d}} . P<0.05^{\mathrm{e}}, P<0.01$ vs. control group ${ }^{\mathrm{b}} ; P<0.05^{\mathrm{c}}, P<0.01$ vs. integrated disease model group

HMG disease model and integrated disease model rats significantly increased plasma FSH concentration $(P<0.05$ vs. blank control; Figure 11). Such a response was significantly attenuated by treatment with high-dose, middle-dose and low-dose NO.1 HRYZ treatment ( $P<0.05$ vs. integrated disease model group). Tamoxifen group and Rupisanjie group had no significant difference. 


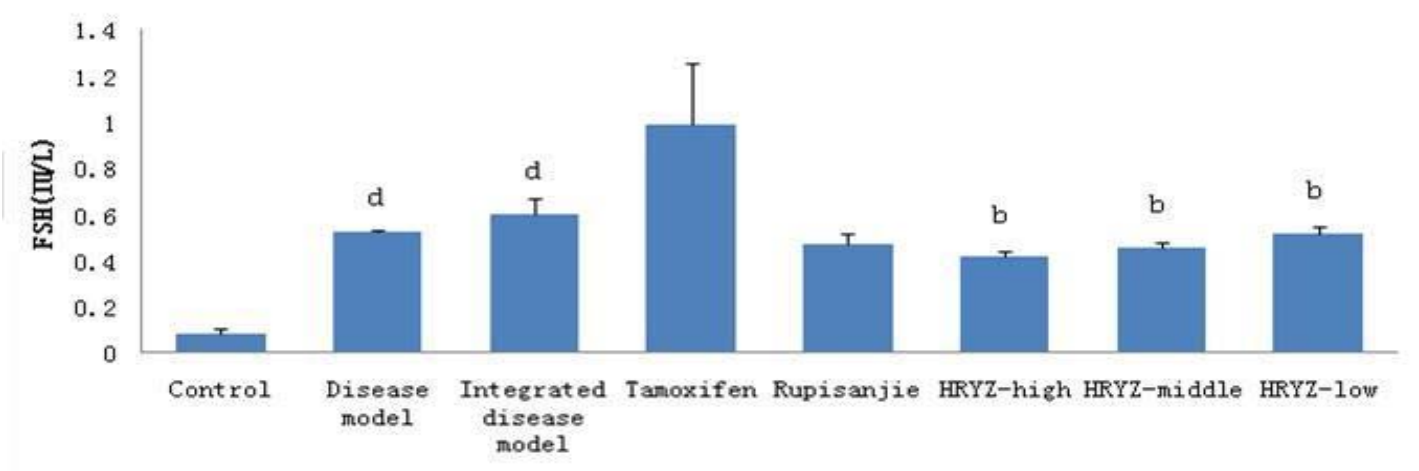

Figure 11: Serum FSH concentration in different groups ${ }^{\mathrm{d}} . P<0.05^{\mathrm{e}}, P<0.01$ vs. control group ${ }^{\mathrm{b}} ; P<0.05^{\mathrm{c}}, P<0.01$ vs. integrated disease model group

LH of HMG disease model and integrated disease model rats had no significant difference compared with control group in our study (Figure 12).

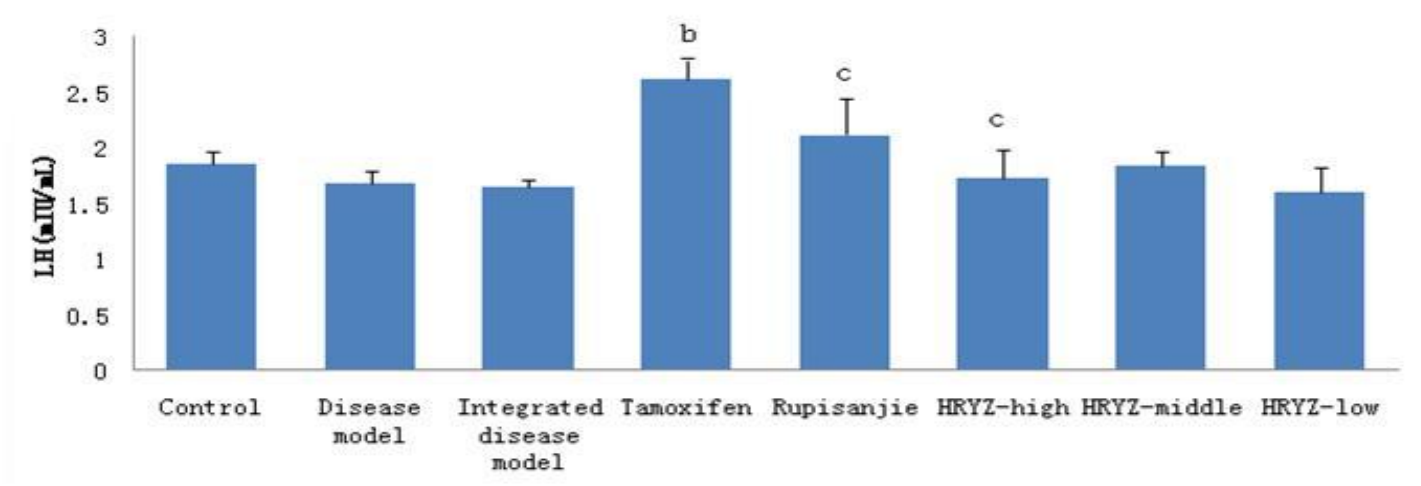

Figure 12: Serum LH concentration in different groups ${ }^{\mathrm{d}}$. $P<0.05^{\mathrm{e}}, P<0.01$ vs. control group ${ }^{\mathrm{b}} ; P<0.05^{\mathrm{c}}, P<0.01$ vs. integrated disease model group

HMG disease model and integrated disease model rats significantly increased GnRH concentration $(P<0.05$ vs. blank control; Figure 13). Such a response was remarkably attenuated by treatment with high-dose NO.1 HRYZ ( $P<0.01$ vs. integrated disease model group) and middle-dose NO.1 HRYZ ( $P<0.05$ vs. integrated disease model group). Tamoxifen group, Rupisanjie group and low-dose NO.1 HRYZ had no significant difference.

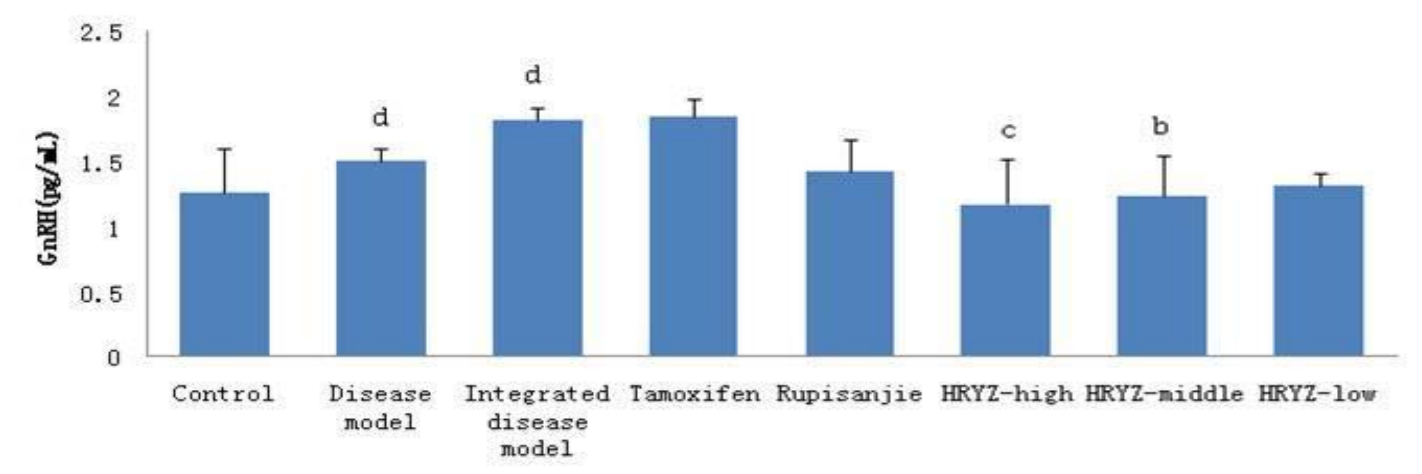

Figure 13: Serum GnRH concentration in different groups ${ }^{\mathrm{d}}$. $P<0.05^{\mathrm{e}}, P<0.01$ vs. control group ${ }^{\mathrm{b}}$; $P<0.05^{\mathrm{c}}, P<0.01$ vs. integrated disease model group

HMG disease model ( $P<0.05$ vs. blank control; Figure 14$)$ and integrated disease model $(P<0.01$ vs. blank control) rats significantly increased 5-HT concentration. Such a response was significantly attenuated by treatment with Rupisanjie, 

http://dx.doi.org/10.4314/ajtcam.v10i4.5

high-dose NO.1 HRYZ ( $P<0.05$ vs. integrated disease model group) and middle-dose NO.1 HRYZ, low-dose NO.1 HRYZ $(P<0.01$ vs. integrated disease model group). Tamoxifen group had no significant difference.

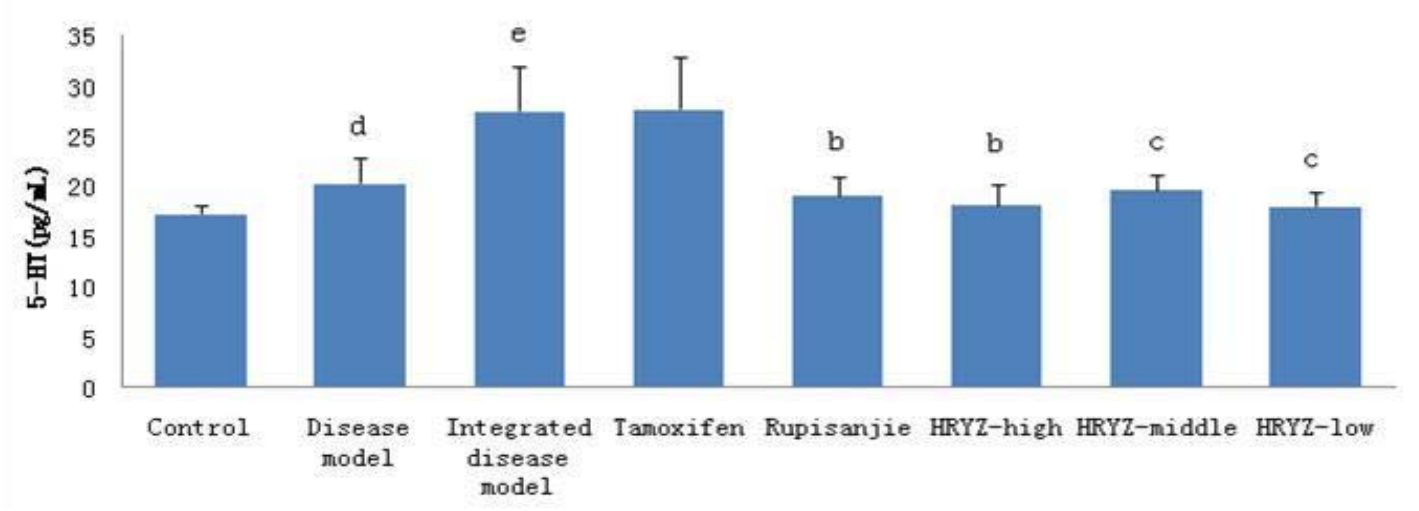

Figure 14: Serum 5-HT concentration in different groups ${ }^{\mathrm{d}}$. $P<0.05^{\mathrm{e}}, P<0.01$ vs. control group ${ }^{\mathrm{b}} ; P<0.05^{\mathrm{c}}, P<0.01$ vs. integrated disease model group

\section{Effect of NO.1 HRYZ on pathomorphology of mammary gland tissue in HMG model rats}

Mammary gland section of normal control rats is shown in Figure 15A. Intense hyperplasia of gland alveolus and lobule, much secretion, vessel arborisation, lymphoplasia and plasma cells were observed in mammary gland of the disease model group and the integrated disease model group (Figure 15B and D). The HMG was still serious in the disease model own control group rats and the integrated disease model own control group rats which were only administrated with water for 30 days, illustrating that HMG model rats did not improve and recover (Figure 15C and E). In tamoxifen-treated group and Rupisanjie-treated group at week 4, the hyperplasia of lobules and gland alveolus of mammary gland lessened, secretion and lymphocyte decreased in intracavitary (Figure 15F and G). The mammary gland of groups given high-dose NO.1 HRYZ and middle-dose NO.1 HRYZ recovered well. There were few hyperplasia in gland alveolus and lobules of mammary gland, and only few secretion and lymphocyte was seen (Figure 15H and I). The low-dose NO.1 HRYZ had no significant effect, the obvious gland alveolus and lobules hyperplasia were seen, and there was much secretion (Figure 15J). These illustrated that NO.1 HRYZ had obvious curative effect in treating HMG, which was even better than positive drugs tamoxifen and Rupisanjie. 

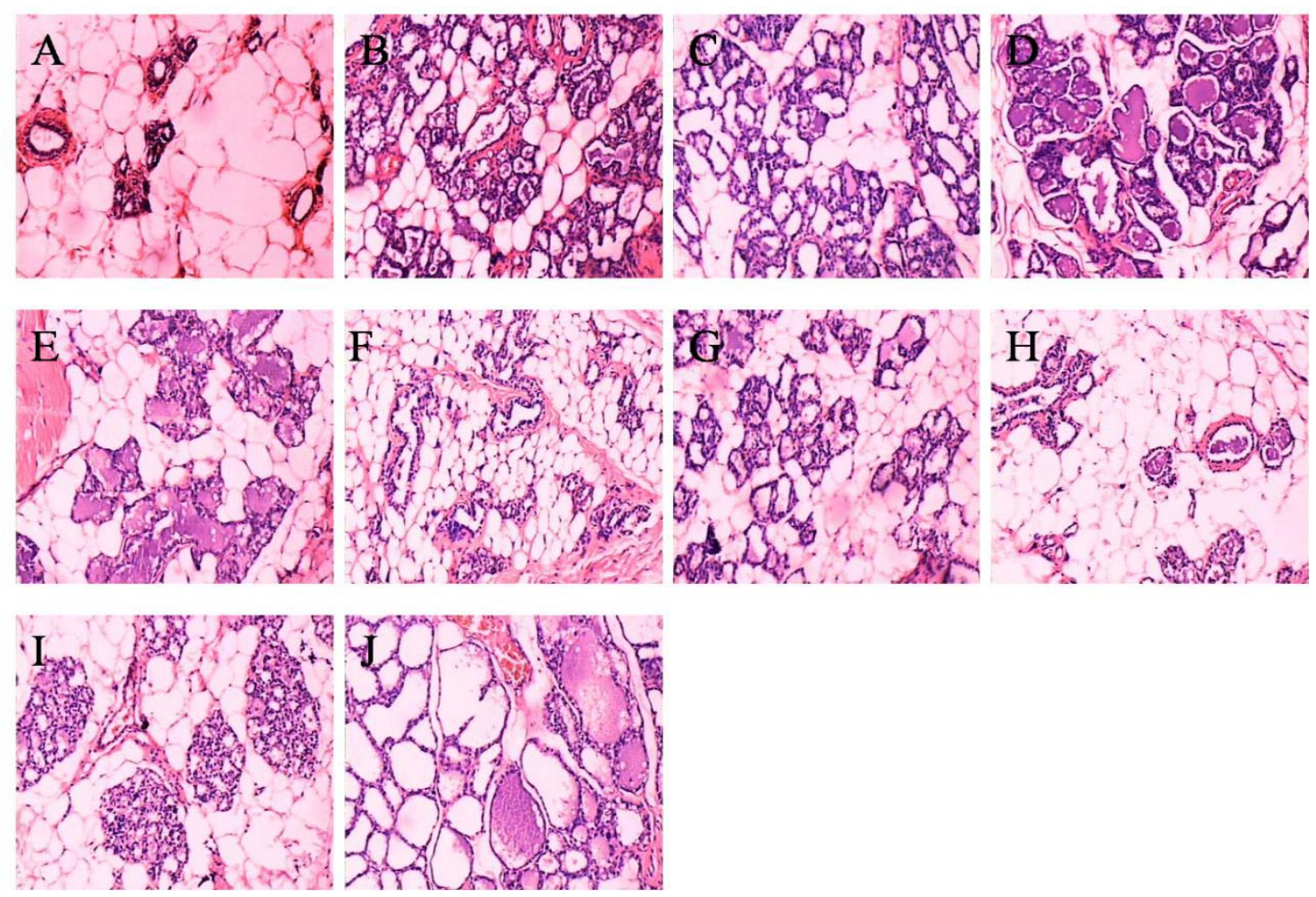

Figure 15: Morphology of mammary gland tissues in each group were observed by microscope (original magnification, $100 \times$ ). (A) blank controls; (B) disease model group; (C) disease model group (own control); (D) integrated disease model group;(E)integrated disease model group(own control); (F) tamoxifen group; (G) Rupisanjie group; (H) high-dose NO.1 HRYZ; (I) middle-dose NO.1 HRYZ;(J)low-dose NO.1 HRYZ

\section{Toxicity test result}

In test one, after administration with $43.1 \mathrm{~g} / \mathrm{kg}$ (200 times of adult dosage) of 24 hours, five mice were found dead and no deaths in later consecutive seven days. And no mice were found dead and no deaths in later consecutive seven days given $34.9 \mathrm{~g} / \mathrm{kg}$ (162 times of adult dosage). In test two, no immediate toxicity was found and no delayed toxicity reaction was found in later 14 days after administration. All mice kept good condition and remained alive. The mice maximum tolerated dose of NO.1 HRYZ was $38.8 \mathrm{~g} / \mathrm{kg}$ freeze-dried powder (180 times of adult dosage). It is the conclusion of this study that NO.1 HRYZ has no side effect on human body and could be used safely in the conventional dose.

\section{Discussion}

This study provides evidence that NO.1 HRYZ acts as a hormone-regulating agent in hyperprolactinemia and HMG model rats. Hypothalamus-pituitary-gonadal axis is one of the constitutions of NIM network and plays an important role in the maintenance of homeostasis of the internal environment and the normal physiological function of the organic body (Goya, R.G.,1991). When extraneous factors act on human body, and sex hormone secretion becomes abnormal, it leads to many kinds of diseases such as hyperprolactinemia, HMG, hysteromyoma, infertility and so on.

The sex hormone disorder occurs in hyperprolactinemia and HMG rat models. NO.1 HRYZ regulated PRL, E2, P, FSH and LH, by adjusting the balance of hypothalamus-pituitary-gonadal axis, resumes the function of ovary, and eliminates the hyperplasia of lobules and gland alveolus. We found PRL was significantly decreased, and $\mathrm{E}_{2}, \mathrm{P}, \mathrm{FSH}$ and LH were increased by NO.1 HRYZ (16.55 g/kg) treatment compared with hyperprolactinemia model group, thus returning to normal. NO.1 

http://dx.doi.org/10.4314/ajtcam.v10i4.5

HRYZ inhibits chronic proliferative inflammation processes with a dose-dependent inhibition of granuloma formation in rat, and also has protective and therapeutic effects on HMG rats induced by oestrogen and progestogen. After injection of oestrogen and progestogen, results showed that E2, PRL, FSH, GnRH and 5-HT were remarkably decreased and P concentration was significantly increased by NO.1 HRYZ $(31.6 \mathrm{~g} / \mathrm{kg}$ or $15.8 \mathrm{~g} / \mathrm{kg})$ treatment compared with HMG model group. Histopathologic examination of NO.1 HRYZ $(31.6 \mathrm{~g} / \mathrm{kg})$ treatment remarkably alleviated the degree of HMG, number of lobules, number of acinars and lobule volumes decreased in different degrees. Therefore, the mechanism of NO.1 HRYZ in treating HMG is by way of regulating endocrinal and immune functions to balance hormonal levels in the body to inhibit the pathological proliferation of mammary gland. In our experiment, we found that the serum PRL increased and $\mathrm{E}_{2}, \mathrm{P}, \mathrm{FSH}$ and LH decreased in hyperprolactinemia rat models. And the serum PRL, E2, FSH, GnRH, 5-HT increased and P decreased in HMG rat models. Although the hormone disorders were different, NO.1 HRYZ could regulate the hormone disorder of hyperprolactinemia and HMG model rats, illustrating that Chinese medical herbs had effective balance function. NO.1 HRYZ could regulate the disorder of hypothalamus-pituitary-gonadal axis in hyperprolactinemia and HMG model rats and could make sex hormones return to normal, thus treating them effectively.

NO.1 HRYZ was composed of ten Chinese herbal medicines, and the chemical constituents varied. In Chinese traditional medicine, drug dose was used very accurately for best curative effects. If there is under-dose in one kind of Chinese medicine, , curative effect is poor; if excessive dosage, toxic reaction or therapeutic change is not usually obvious. In our experiment, we set high-dosage, middle-dosage and low-dosage of NO.1 HRYZ in searching for the best dosage. At last, we found that middle-dosage of NO.1 HRYZ produced the best result. We thought that there might be some chemical reactions in high-dosage NO.1 HRYZ for reducing effect, so high-dose of NO.1 HRYZ has less effect on E2 concentration than middle-dose group. The middle-dosage of NO.1 HRYZ acted the best curative effect, so high-dose group and middle-dose group seem to have same effects on P, FSH and LH even with the increased dosage of NO.1 HRYZ. The specific reasons are still being studied by our researchers.

In mammals, PRL can regulate mammogenesis, promote milk production, initiate and sustain lactation through autocrime and paracrime as a kind of cell factor (Yu et al. , 1998; Freeman et al., 2000; Kelley et al., 2007). PRL produces a variety of effects on breast tissue including influences on milk protein synthesis, uridine conversion and incorporation into DNA, mammary cell sodiumt-ransport, and breast fatty acid synthetase activity (Malarkey et al., 1977). However, relevant reports about the relationship between hyperprolactinemia and HMG were not found. In our experiment, the PRL concentration was determined in the hyperprolactinemia rat serum, and we found the PRL was much more than control rats. Meanwhile, hyperplasia was found in the mammary gland of hyperprolactinemia model rats. Compared with control group, the serum PRL of HMG model rats increased significantly. These investigations demonstrated that there was close relationship between hyperprolactinemia and HMG, and hyperprolactinemia promoted the formation of HMG. Previously, doctors thought that HMG was induced by excessive E2 and insufficient $\mathrm{P}$ in patient serum, and ignored the action and importance of PRL on mammary gland (Shi and Zhou, 2010). Our research revealed that PRL played a very important role in the formation of HMG, and hyperprolactinemia promoted the formation of HMG. However, the exact mechanism of PRL in the action of HMG is not clear. Although we only studied the hormone and morphology of mammary gland tissue in present experiment, more experiments such as hormone receptor and gene study between the two diseases are being performed by our researchers deeply.

In conclusion, the results presented significant therapeutic effects of NO.1 HRYZ by influencing sex hormone levels. Hyperprolactinemia is closely correlated with HMG, and hyperprolactinemia promotes the formation of HMG. NO.1 HRYZ could be used for hyperprolactinemia and HMG treatment effectively. Further investigation about its real bioactive compounds is being studied by our researchers.

\section{Acknowledgements}

This project was supported by the scientific research fund project of Wuhan Municipal Health (WZ11C07). 


\section{References}

1.Biller BM, Luciano A and Crosignani PG.(1999).Guidelines for the diagnosis and treatment of hyperprolactinemia. J Reprod Med, 44, 1075-84.

2.Bennett I. C., Cufffrey, Mc and Mccattrey, E.(1990). Serum oestradiol in women with and without breast disease,Br J Cancer, 61,142-147.

3. Fallois JV, Etterii-Billenkamp, U. and Sehindler, E.M. (1995). Danazol for treatment of fibrocystic mastopathy. Zentralbl Gynakol, 117(9), 457-465.

4.Freeman ME,Kanyicska B and Lerant A.(2000). Prolactin:structure,function, and regulation of secretion.Physiol Rev, 80(4):1523-1526.

5.Gerhard Lutz.(2012). Hair loss and hyperprolactinemia in women.Endocrinology, 4(1):73.

6.Greer ME, Moraczewski, T and Rakoff, JS.(1980). Prevalence of hyperprolactinemia in anovulatory women. Obstet Gynecol, 56, 65-9.

7.Jia, CM.(2011).The cause analysis of hyperplasia of mammary glands. Chin J of Clinical Rational Drug Use, 4(3A):157-158.

8. Lin, K.C., Kawamura, N. and Okamura, H. (1988). Inhibition of ovalation,steroidogenesis and collagenolytic activity in rabbitts by sulpiride-induced hyperprolactinemia. J.Reprod Fert, 83:612.

9.Kelley KW, Weigent, DA and Kooijman, R.(2007). Protein hormones and immunity. Brain Behav Immun,21(4):384-392. 10.Lee, D-Y, Oh, K-L and Yoon B-K. (2012). Prevalence of hyperprolactinemia in adolescents and young women with menstruation-related problem. Am J Obstet Gynecol, 206, 213.e1-5.

11.Lu, CL.(2004). Diagnosis and treatment of nipple discharge. Acta Medicinae Sinica, 17(3):454.

.12.Mah, PM and Webster J.(2002).Hyperprolactinemia:etiology,diagnosis and management.Semin Reprod Med,20(4),365-374.

13.Malarkey, WB, Schroeder, LL and Stevens, VC.(1977).Disordered nocturnal prolactin regulation in women with breast cancer.Cancer Res,37(12):4650.

14.Milliken, EL, Ameduri, RK and Landis, MD.(2002). Ovarian hyperstimulation by LH leads to mammary gland hyperplasia and cancer predisposition in transgenic mice.

Endocrinology, 143(9), 3671-3680.

15.Rao, JC, Li LZ and Chen YS.(1992).The preparation and pathological type of HMG animal model. Chinese Journal of Pathophysiology, 8(6):671.

16. Goya, R.G..(1991).The immune-neuroendocrine homeostatic network and aging, Gerontology, 37, 208-213.

17.Shi XC, Zhou SP.(2010).Clinical Research on Mammary Hyperplasia Treated with Integrated Chinese and Western Medicine. World Journal of Integrated Traditional and Western Medicine,5(12):1063.

18.Tan, CE, Wang, JP and Contantino, JP.(2003).Effect of tamoxifen on benign breast disease in women at high risk for breast cancer. J Natl Cancer Inst, 95(4), 302-307.

19.Wang LS, Zhao DQ and Lin D.(2011).The anti-hyperplasia of mammary gland effect of Thladiantha dubia root ethanol extract in rats reduced by estrogen and progestogen. Journal of Ethnopharmacology, 134, 136-140.

20.Webster, J, Piscitell, G and Polli, A.(1994).A Comparison of Cabergoline and Bromocriptine in the Treatment of Hyperprolactinemic Amenorrhea. N Engl J Med,

331,904-909.

21.Xu, KY.(2011).The etiology and clinical intervention of hyperplasia of mammary glands.China Prac Med,6(28):99.

22.Yu-lee, LY, Luo GY and Book ML.(1998). Lactogenic hormone signal transduction

[J].Biol Reprod, 58(2):295-301. 